\title{
New Regeneration Process of Heavy-Metal-Loaded Chelating Resins
}

\author{
By Philippe Menoud, Laurent Cavin, and Albert Renken*
}

An alternative to the classical acid-base regeneration of chelating resins loaded with heavy metals is investigated. The new process consists in recovering the heavy metals with recyclable soluble complexing agents. The semiclosed reactor includes a fixed bed and a stirred tank. A three-parameter model, which implies a double equilibrium in series, is introduced. When less than $10 \%$ of the metal is still fixed on the resin at the end of the desorption, a simplified form of the model with two parameters, which describes a quick equilibrium followed by a first-order kinetics reaction, is proposed. Both forms of the model can simulate results for different experimental conditions (polymer and metal types, polymer initial concentration). It was observed for both cases that the first equilibrium constant depends on the polymer type and that the rate constant of desorption depends on the metal type. The scale-up of desorption is then conducted at mini-pilot scale. Scale-up criteria tend to minimize the desorption time and the soluble polymer quantity used.

\section{Introduction}

Adsorption to chelating resins is a common method to recover heavy metals from wastewater containing very small concentrations of heavy metals. The main advantage of the chelating resins compared to classical ion exchangers is their ability to form strong complexes with heavy metals. The stability of the complexes is very well described by adsorption constant $K$ [1]. A favorable adsorption, with a high adsorption constant, implies indeed that it will be difficult to detach the metal from the resin in the regeneration step.

Classical resin regeneration is based on the $\mathrm{pH}$ dependence of the adsorption constants, which tend to decrease by lowering the $\mathrm{pH}$. An acidic solution can solubilize the heavy metal and the resin is then regenerated under alkaline conditions to perform a further metal adsorption. Both acid and base are used in excess quantities. It was shown [2] for the system $\mathrm{Cu}^{2+} / \mathrm{Chelamine}$ (see Materials and Methods) that a molar ratio $\mathrm{Cu}^{2+} / \mathrm{H}_{2} \mathrm{SO}_{4} / \mathrm{NaOH}$ of $1 / 5 / 4$ is required to perform a full desorption/regeneration of the resin. The regeneration process leads therefore to a consumption of acid and bases and thus a considerable production of salts.

In order to avoid this consumption of chemicals and salt production, a new recovery method of the metallic ion by use of recyclable chemicals was developed [3]. The idea is to solubilize the heavy metal with soluble strong complexing agents (for example ethylenediamine tetraacetate, EDTA). The so-formed metallic complex is electrolyzed in a further step and the complexing agent is recycled for a further regeneration cycle.

The main problems to overcome are:

- loss of the complexing agent by degradation

- dilution of the complexing agent solution due to washing processes

[*] Dr. P. Menoud, Colors 8.1, Ciba Specialty Chemicals, CH-1870 Monthey; Dipl. Chem.-Ing. L. Cavin, Prof. Dr. A. Renken (author to whom correspondence should be addressed), LGRC, DC, EPFL, CH-1015 Lausanne, Switzerland.
The solution needs to be reconcentrated by a membrane process (see Fig. 1). The use of high-molecular-weight complexing agents, like soluble chelating polymers, greatly facilitates the concentration step: reverse osmosis, used for the separation of small molecules, like metal complexes of EDTA, can be replaced by an ultrafiltration module.

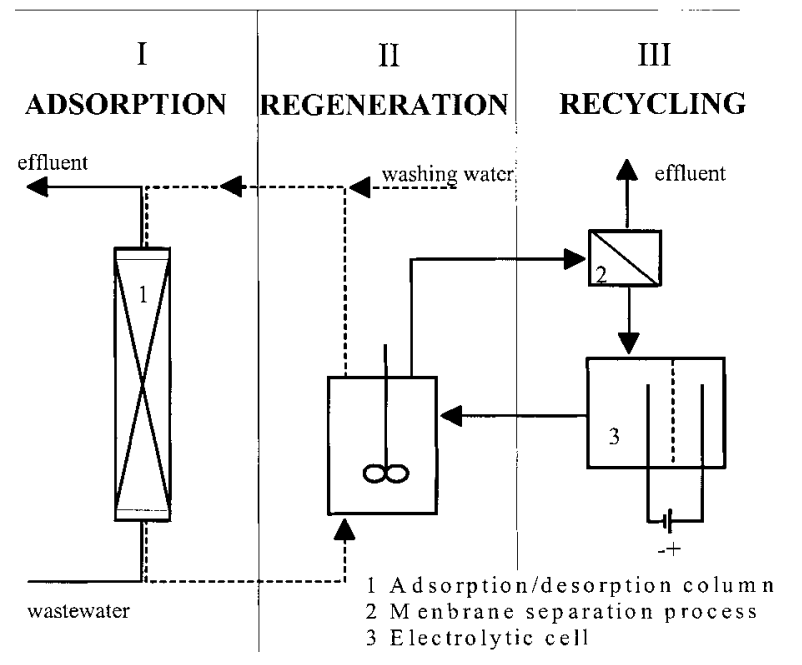

Figure 1. Global metal recovery process.

Several researchers investigated the feasibility of desorption of loaded resins with strong soluble complexing agents. Bolto et al. [4] proposed a regeneration process of weak-acid resins loaded with cadmium with EDTA or nitrilotriacetate (NTA). Tipnis and Dasare [5] compared different desorptions of resins loaded with mercury. The entire mercury picked up by weakly basic anion exchangers was eluted by $0.5 \mathrm{~N}$ or $1.0 \mathrm{~N}$ nitric acid solutions. Compared to nitric acid, EDTA buffered at $\mathrm{pH} 10$ appears to be the best eluting agent for mercury. However, none of these authors tried to describe the kinetic characteristics of the desorption process with strong complexing agents.

The purpose of this work is to study the regeneration of a loaded resin with different strong complexing agents. A kinetic model will be developed in order to understand the 
fundamentals of this regeneration process, especially the influence of the molecular size of the complexing agents on the desorption.

\section{Materials and Methods}

\subsection{Chelating Resin}

The resin used for this application was the Chelamine Standard (JPS-Chimie, Neuchâtel, Switzerland). It was synthesized using a polyacrylamide gel with tetraethylene pentamine chelating groups. Before each use, the resin was treated in a fixed bed with a solution of sulfuric acid ( $\mathrm{pH} 1)$, then washed with demineralized water. In a further step the resin underwent a regeneration with a sodium hydroxide solution ( $\mathrm{pH} 11.5)$ and a wash with demineralized water to a defined $\mathrm{pH}\left(\mathrm{pH}_{0}\right)$. If not mentioned otherwise, $p H_{0}$ was equal to 9.5 .

The mean radius of the resin particles $r_{p}$ was measured by laser diffraction (Particle Sizer 2600 E, Malvern Instruments, England) and was $1.73010^{-4} \mathrm{~m}$ for unloaded Chelamine and $1.52410^{-4} \mathrm{~m}$ for the Chelamine loaded with $\mathrm{Cu}^{2+}$. The porosity of the fixed bed was determined by the measurement of the residence-time distribution of a void volume marker. $\beta-\mathrm{D}(+)$ glucose (Sigma, St-Louis, USA) was used to measure the total void volume in the fixed bed. The global porosity of a fixed bed of Chelamine was estimated to a value of $\varepsilon_{r}=0.87$.

\subsection{Complexing Agents and Adsorbate}

The soluble polymers were the polyacrylic acid $(P A A)$ and the polyethyleneimineacetic acid $(P E I A C)$. The $P A A\left(M_{w}=\right.$ $20^{\prime} 000 \mathrm{~g} \mathrm{~mol}^{-1}$ ) was purchased from Fluka AG (Buchs, Switzerland) and was used without prior treatment. The PEIAC $\left(M_{w}=5^{\prime} 000\right.$ and $\left.750{ }^{\prime} 000 \mathrm{~g} \mathrm{~mol}^{-1}\right)$ was synthesized by Dr. G. Jeanneret-Gris (JPS Chimie, St-Aubin, Switzerland) from polyethyleneimine (Aldrich, USA) according to Geckeler et al. [6]. It was purified by ultrafiltration according to a method described by Menoud [7]. All sulfates of hydrated metallic cations (purity: purum p.a.) used in this work were purchased from Fluka AG. The absorbance of the complexes $\mathrm{PM}^{2+}$ was measured by a UV-VIS spectrophotometer (Diode Array HP 8452A, Hewlett Packard, Palo Alto, USA). The measured absorbance was transformed into concentration according to the Lambert-Beer's law: extinction coefficients $\varepsilon_{P M, \lambda}$ obtained by an appropriate calibration are given in Tab. 1 .

\subsection{Experimental Procedure}

The regeneration installation included a fixed-bed column and a continuous stirred tank reactor (CSTR) as depicted in Fig. 2. A pump located between the fixed-bed column and the CSTR ensured a constant flow rate $Q$. A pH-stat (Impulso-
Table 1. Wavelengths and molar absorption coefficients for heavy metal complexes.

\begin{tabular}{|c|c|c|c|}
\hline Polymer & Metal & $\begin{array}{c}\lambda \\
{[\mathrm{nm}]}\end{array}$ & $\begin{array}{c}\mathcal{E}_{P M, \lambda} \\
{\left[\mathrm{M}^{-1} \mathrm{~cm}^{-1}\right]}\end{array}$ \\
\hline \multirow[t]{2}{*}{$P A A 20^{\prime} 000$} & $C u^{2+}$ & 690 & 42.03 \\
\hline & $N i^{2+}$ & 398 & 8.90 \\
\hline \multirow[t]{2}{*}{ PEIAC 25'000 } & $C u^{2+}$ & $\begin{array}{l}582 \\
686 \\
\end{array}$ & $\begin{array}{l}43.00 \\
87.40 \\
\end{array}$ \\
\hline & $\mathrm{Ni}^{2+}$ & $\begin{array}{l}582 \\
686 \\
\end{array}$ & $\begin{array}{r}10.44 \\
4.05 \\
\end{array}$ \\
\hline$P E I A C 750^{\circ} 000$ & $\mathrm{Ni}^{2+}$ & 578 & 11.06 \\
\hline \multirow[t]{2}{*}{ EDTA } & $\mathrm{Cu}^{2+}$ & $\begin{array}{l}382 \\
732 \\
\end{array}$ & $\begin{array}{r}4.26 \\
93.83 \\
\end{array}$ \\
\hline & $N i^{2+}$ & $\begin{array}{l}382 \\
732\end{array}$ & $\begin{array}{r}13.26 \\
1.94\end{array}$ \\
\hline
\end{tabular}

mat, Dosimat, pH-Meter, Metrohm, Herisau, Switzerland) adjusted the $\mathrm{pH}$ in the CSTR to a defined constant $\mathrm{pH}$ (the "work pH") with adjunction of an $\mathrm{NaOH}$ solution. The UVVIS spectrophotometer measured the on-line concentration of the complex $\mathrm{PM}^{2+}$ at the outlet of the CSTR. The column and the UV-VIS cell were thermostatted at $20^{\circ} \mathrm{C}$. Two scales of equipment were used for these experiments: the laboratoryscale apparatus (column diameter $d_{t}=0.015 \mathrm{~m}$ ) and the pilot plant $\left(d_{t}=0.052 \mathrm{~m}\right)$.

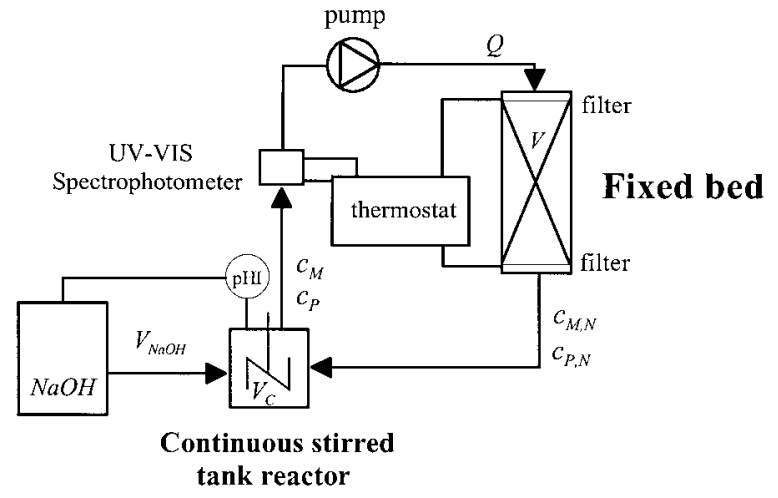

Figure 2. Schematic diagram of experimental apparatus

The fixed-bed column was filled with a quantity of resin $m$. The resin was loaded to saturation with a concentrated metal solution $\left(c_{0}=5 \mathrm{~kg} \mathrm{~m}^{-3}\right)$ and washed with distilled water. The CSTR contained a volume $V_{C o}$ of a polymer solution with a concentration $c_{P, 0}$. At the beginning of the regeneration (time $t=0$ ), the on-line UV-VIS measurement, the pump and the $\mathrm{pH}$-stat were switched on. The constancy of the flow rate was checked and the volume of the added $\mathrm{NaOH}$ solution $\mathrm{V}_{\mathrm{NaOH}}$ was recorded. The regeneration was over when the absorbance at the outlet of the CSTR reached a constant value. In case of partial metal desorption, the heavy metal remaining on the resin was desorbed with a solution $\left(100 \mathrm{~mol} \mathrm{~m}^{-3}\right)$ of the strong ligand EDTA and washed with distilled water for a further regeneration. 
The determination of the initial concentration of metal on the resin $q_{0}$ and the polymer capacity $q_{P}$ was determined by a method described by Menoud [2].

For all laboratory experiments, $0.4 \mathrm{~g}$ of resin was used (17.05 $\mathrm{g}$ for the pilot plant). As the volume of the fixed bed increased by a factor 2 when metal was totally desorbed from the resin, an average value of the reactor volume $V=2.42510^{-6} \mathrm{~m}^{3}$ was used (large scale: $1.11510^{-4} \mathrm{~m}^{3}$ ).

The initial volume of the CSTR $V_{C, 0}$ was $7.510^{-5} \mathrm{~m}^{3}$ (large scale: $2.52810^{-3} \mathrm{~m}^{3}$ ). The dead volume $V_{M}$ included the volumes of the pipes and of the liquid at the top of the fixed bed and was $3.0510^{-6} \mathrm{~m}^{3}$ (large scale: $2.5010^{-4} \mathrm{~m}^{3}$ ).

The molar ratio $\gamma$ between the initial free sites of the complexing agent and the initial metallic cations present on the resin in the desorption reactor is given by ${ }^{1)}$ :

$\gamma=\frac{c_{P, 0} V_{C, 0}}{m q_{0}}$

\section{Theoretical Basis and Model}

\subsection{Reactor Model}

The final process consists of a fixed bed modeled by a cascade of $N$ ideal continuous stirred tanks, a dead volume and a CSTR. The value of $N$ is determined from a residence-time distribution and is found to be equal to 3 .

At the beginning of the desorption process, the viscous polymer solution was only slowly mixed with the low-viscous medium. This led to the formation of dead volume near the walls and channeling. Therefore, an initial by-pass $r=0.3$ ( $r=0.1$ for the pilot plant) was included in the model [2].

\subsection{Kinetic Model}

A loaded chelating resin $\left(R M^{2+}\right)$ is desorbed by a chelatingsoluble polymer $(P)$. A kinetic model and a simplification of this model is developed in this section. Simulation results with a diffusion model show that pore diffusion and external mass transfer are not rate-limiting for all chemicals participating in the reaction $\left(P, P M^{2+}\right.$ and co-ions like $\left.\mathrm{Na}^{+}\right)$. The kinetics is governed by the desorption reaction on the active sites of the resin. The kinetic model includes a double equilibrium as follows (the concentrations of each species is given on the second line):

$$
\begin{array}{cccc}
R M^{2+}+P \frac{k_{1}}{\overparen{k_{-1}}} & \left\{R M^{2+} P\right\} & \frac{k_{2}}{\overparen{k_{-2}}} & R+P M^{2+} \\
\left(q_{i}-q_{c, i}\right) c_{P, i} & q_{c, i} & & \left(q_{0}-q_{i}\right) c_{M, i}
\end{array}
$$

The loaded resin reacts with the soluble polymer and forms an intermediate complex $\left(\left\{R M^{2+} P\right\}\right)$ which splits into a complex $\left(P M^{2+}\right)$ and an unloaded resin site $(R)$. The same

1) List of symbols at the end of the paper. type of kinetics is encountered in inorganic chemistry for the substitutions in octahedral complexes in solution, described by the Eigen-Wilkins mechanism [7]. The first step corresponds to the formation of a pair of ions: this step is not depending on the nature of the metallic cation. The second step is an exchange of a molecule of water with a molecule of ligand. This step depends on the nature of the metallic cation. The concentration $c_{P, i}$ represents the concentration of free polymer sites in the tank $i$ of the fixed bed. The first equilibrium is assumed to be reached much faster than the second one, one can thus write:

$\frac{d q_{c, i}}{d t}=k_{1}\left(q_{i}-q_{c, i}\right) c_{P, i}-k_{-1} q_{c, i}$

Assuming a thermodynamic equilibrium the concentration of the intermediate $\left\{R M^{2+} P\right\}$ is given by:

$q_{c, i}=\frac{K_{1} c_{P, i} q_{i}}{1+K_{1} c_{P, i}}$

with the first equilibrium constant:

$K_{1}=\frac{k_{1}}{k_{-1}}$

The second step is described by the following equation:

$\frac{d q_{c, i}}{d t}=\frac{d q_{i}}{d t}=-k_{2} q_{c, i}+k_{-2}\left(q_{0}-q_{i}\right) c_{M, i}$

A combination of (4) and (6) gives the kinetic equation for the model:

$\frac{d q_{i}}{d t}=-k_{2}\left(\frac{K_{1} c_{P, i}}{1+K_{1} c_{P, i}}\right) q_{i}+k_{-2}\left(q_{0}-q_{i}\right) c_{M, i}$

A simplification of the model is obtained when the constant $k_{-2}$ is sufficiently small to be neglected. The simplified model postulates that the desorption goes on as long as loaded resins $R M^{2+}$ and free polymers sites $P$ are available. The desorption of loaded resin through a polymer is then:

$\frac{d q_{i}}{d t} \cong-k_{2}\left(\frac{K_{1} c_{P, i}}{1+K_{1} c_{P, i}}\right) q_{i}$

This behavior is sometimes called "saturation kinetics". Its mathematical representation is a hyperbola. For a high concentration of soluble polymer $\left(K_{1} \mathrm{c}_{P, i}>>1\right)$, the desorption rate is simplified to a first-order rate independent from the polymer concentration:

$\frac{d q_{i}}{d t} \cong-k_{2} q_{i}$

A second-order kinetics describes the situation at low polymer concentrations $\left(K_{1} \mathrm{c}_{P, i}<<1\right)$ :

$\frac{d q_{i}}{d t} \cong-k_{2} K_{1} c_{P, i} q_{i}$

Numerical solutions of the differential equations for the different models developed were obtained with the software Simusolv (The Dow Chemical Company, Midland, Michigan, USA). The integration method used was a Gear algorithm 
(BDF-DIFSUB) with variable step and order. Kinetic parameters for the models were estimated by curve fitting.

\section{Results and Discussion}

A wide range of experimental parameters can affect the desorption of metal ions with a soluble polymer, for example:

- the residence time $\tau$ in the fixed bed

- the $\mathrm{pH}$ of the polymer solution

- the molar ratio $\gamma$

- the type of metal adsorbed on the resin

- the type and the molecular weight of soluble polymer

Preliminary experiments studying the effects of the residence time $\tau$ in the fixed bed and the $\mathrm{pH}$ of the polymer solution lead to the following conclusions:

- the loaded soluble polymer precipitates at low $\mathrm{pH}$ solutions $(\mathrm{pH}<5.3)$.

- a high $\mathrm{pH}$ involves a large consumption of $\mathrm{NaOH}$ for the preparation of the alkaline solutions and precipitation of the metal ions as hydroxide.

Thus, an intermediate $\mathrm{pH}$ value of 7.3 was selected. In order to avoid any gradient of $\mathrm{pH}$ the residence time in the fixed bed has to be low compared to the characteristic desorption time [2].

\subsection{Influence of the Soluble Polymer Concentration}

Fig. 3 describes several desorptions of the $\mathrm{Ni}^{2+}$-loaded resin Chelamine with the PEIAC 25'000. The experimental conditions for these desorptions are given in Tab. 2. The evolution of the reaction is given by the elution volume, $V_{E}\left(V_{E}=Q t\right)$ and the ordinate indicates the concentration of metallic complex $c_{M}\left[\mathrm{~mol} \mathrm{~m}^{-3}\right]$ in the CSTR. The effect of the initial polymer concentration $c_{P, 0}$ (corresponding to changing $\gamma$ ) at $\mathrm{pH} 7.3$ was studied.

The simplified model ( $7 \mathrm{~b}$ ) gives a good representation of the experimental values. The optimized kinetic parameters remain constant from one experiment to the other, $K_{1} \cong$ $0.035 \mathrm{~m}^{3} \mathrm{~mol}^{-1}$ for each run and the average value of $k_{2}=8.0$ $10^{-3} \mathrm{~s}^{-1}$. The value of $K_{1}$ obtained with the complete model is maintained, as this parameter is totally independent of $\left(K_{2}\right.$ $\left.=k_{2} / k_{-2}\right)$

It is important to notice that a $\gamma$ lower than 1 corresponds to a lack of soluble polymer. REG124 is such a run, from which the capacity of the complexing agent can be determined $q_{P}=2.076 \mathrm{~mol} \mathrm{~kg}^{-1}$. An increase in $\gamma$ (and in concentration $c_{P, O}$ ) shortens the desorption time. However, if some polymer is lost during the global recovery process presented in the introduction (during ultrafiltration or electrolysis), it is important to mini-

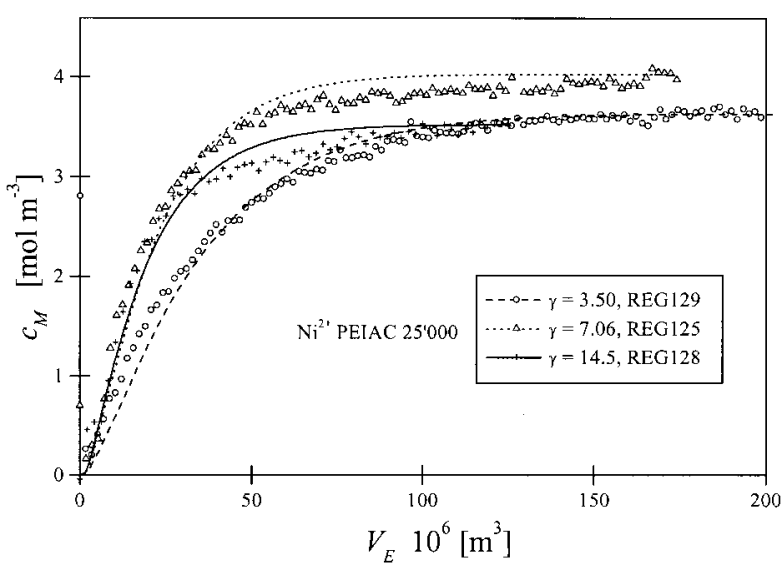

Figure 3. Effect of polymer concentration (PEIAC 25,000) on the desorption of the resin Chelamine loaded with $\mathrm{Ni}^{2+} . m=0.4 \mathrm{~g}, \mathrm{pH}=7.3$, simplified model, (7b)

mize its quantity $(\gamma)$ in the desorption. A value of $\gamma=1$ represents a quantity of polymer just sufficient to desorb all the metal from the resin, but the desorption time is large. For very high values of $\gamma$, the equation can be further simplified and (8) used.

\subsection{Desorption Behavior for Different Types of Metal and Soluble Polymer}

First, the desorptions of two different heavy-metal ions with $P E I A C 25^{\prime} 000$, and then with PAA 20'000 are compared to determine the influence of the type of metal. Then the desorptions of $\mathrm{Ni}^{2+}$ with two different soluble polymers provide information on the influence of the type of soluble polymer. Finally, the desorptions of $\mathrm{Ni}^{2+}$ with PEIAC of two molecular masses gives the influence of the molecular mass on the desorption. The various desorptions and their parameters used for the simulation are summarized in Tabs. 2 and 3 .

It was shown that the desorption of the system PEIAC $25^{\prime} 000 / \mathrm{Ni}^{2+}$ was complete (REG129). Fig. 4 shows that the desorption with PEIAC 25'000 of a resin loaded with $\mathrm{Cu}^{2+}$ is partial (REG140). The remainder of the metal ions represents $60 \%$ of the initial amount and their quantity is given by $q_{\text {rest }}$ (Tab. 3). An excess of soluble polymer $(\gamma>1)$ does not desorb all the $C u^{2+}$ from the loaded resin. If the stability constant of the complexing agent and heavy metal is high compared to the

Table 2. Overview on the experimental conditions for the desorptions of $\mathrm{Ni}^{2+}$ with $P E I A C 25^{\prime} 000 . \mathrm{pH}=7.3$, $m=0.4 \mathrm{~g}$, simplified model, (7b).

\begin{tabular}{|c|c|c|c|c|c|c|}
\hline $\begin{array}{l}\text { Exp. } \\
\text { REG }\end{array}$ & $\begin{array}{l}Q 10^{7} \\
\mathrm{~m}^{3} \mathrm{~s}^{-1}\end{array}$ & $\begin{array}{c}c_{P .0} \\
\mathrm{~mol} \mathrm{~m}^{-3}\end{array}$ & $\begin{array}{l}q_{0} / q_{\text {rest }} \\
\mathrm{mol} \mathrm{kg}^{-1}\end{array}$ & $\begin{array}{l}\gamma \\
-\end{array}$ & $\begin{array}{c}K_{I} \\
\mathrm{~m}^{3} \mathrm{~mol}^{-1}\end{array}$ & $\begin{array}{l}k_{2} \\
\mathrm{~s}^{-1}\end{array}$ \\
\hline 124 & 0.768 & 2.58 & $0.847 / 0.344$ & 0.59 & 0.035 & $7.610^{-3}$ \\
\hline 129 & 0.691 & 14.45 & 0.805 & 3.50 & 0.035 & $9.010^{-3}$ \\
\hline 125 & 0.888 & 32.48 & 0.898 & 7.06 & 0.035 & $9.610^{-3}$ \\
\hline 128 & 0.835 & $\overline{57.80}$ & 0.775 & 14.54 & 0.036 & $7.210^{-3}$ \\
\hline 131 & 0.937 & 113.80 & 0.882 & 25.20 & 0.035 & $\left(19.210^{-3}\right)$ \\
\hline
\end{tabular}


Table 3. Parameters for regenerations with different soluble polymers and heavy metals. $\mathrm{pH}=7.3, m=0.4 \mathrm{~g}$, except for REG150 $m=17.05 \mathrm{~g}$.

\begin{tabular}{|c|c|c|c|c|c|c|c|c|c|c|}
\hline $\begin{array}{l}\text { Exp. } \\
\text { REG }\end{array}$ & $P / M^{2+}$ & $\begin{array}{r}210^{7} \\
\mathrm{~m}^{3} \mathrm{~s}^{-1} \\
\end{array}$ & $\begin{array}{c}c_{P, Q} \\
\mathrm{~mol} \mathrm{~m}^{-3}\end{array}$ & $\begin{array}{l}\gamma \\
- \\
\end{array}$ & $\begin{array}{c}q_{P} \\
\mathrm{~mol} \mathrm{~kg}^{-1} \\
\end{array}$ & $\begin{array}{l}q_{0} / q_{\text {rest }} \\
\text { mol kg }\end{array}$ & $\begin{array}{c}K_{I} \\
\mathrm{~m}^{3} \mathrm{~mol}^{-1} \\
\end{array}$ & $\begin{array}{l}k= \\
s^{-1} \\
\end{array}$ & $\begin{array}{c}k_{-2} \\
\mathrm{~m}^{3} \mathrm{~s}^{-1} \mathrm{~mol}^{-1} \\
\end{array}$ & $\begin{array}{c}K \\
\mathrm{~mol} \mathrm{~m}_{2} \\
\end{array}$ \\
\hline 101 & $P A A 20^{\prime} 000 / \mathrm{Cu}^{2+}$ & 0.735 & 36.00 & 7.60 & $1.800^{1)}$ & $0.924 / 0.457$ & 0.100 & $1.010^{-3}$ & $3.510^{-4}$ & 3 \\
\hline 113 & $P A A 20^{\prime} 000 / \mathrm{Ni}^{2+}$ & 0.600 & 36.00 & 9.99 & $1.800^{1)}$ & 0.711 & 0.100 & $1.210^{-2}$ & - & - \\
\hline 140 & PEIAC $25^{\prime} 000 / \mathrm{Cu}^{2+}$ & 1.010 & 19.83 & 4.29 & $2.000^{2)}$ & $0.900 / 0.537$ & 0.035 & $2.110^{-3}$ & $7.810^{-4}$ & 3 \\
\hline 147 & PEIAC $750^{\prime} 000 / \mathrm{Ni}^{2+}$ & 0.976 & 1.88 & 0.44 & $1.438^{3)}$ & $0.826 / 0.460$ & 0.010 & $8.010^{-3}$ & - & - \\
\hline 148 & PEIAC $750^{\prime} 000 / \mathrm{Ni}^{2+}$ & 2.179 & 17.72 & 5.04 & $1.438^{3)}$ & $0.686 / 0.090^{4)}$ & 0.010 & $10.610^{-3}$ & $8.710^{-5}$ & 120 \\
\hline 150 & PEIAC $25^{\prime} 000 / \mathrm{Ni}^{2+}$ & 33.95 & 10.38 & 1.96 & $2.076^{5)}$ & $0.784 / 0.065$ & $0.035^{6)}$ & $8.010^{-36)}$ & - & 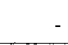 \\
\hline \multirow[t]{2}{*}{143} & PEIAC $25^{\prime} 000 / \mathrm{Ni}^{2+}$ & 0.903 & 33.51 & 8.81 & $2.076^{5)}$ & 0.317 & 0.035 & $8.010^{-3}$ & - & \\
\hline & $/ \mathrm{Cu}^{2+}$ & & & & & $0.425 / 0.308$ & 0.035 & $2.110^{-3}$ & $5.710^{-3}$ & 0.4 \\
\hline
\end{tabular}

stability constant of the resin-metal complex, complete desorption is impossible even at $\gamma>1$.

The desorptions of $\mathrm{Ni}^{2+}$ (REG113) and $\mathrm{Cu}^{2+}$ (REG101) with $P A A 20$ '000, leads to the same conclusions (Tab. 3). The desorption of $\mathrm{Ni}^{2+}$ is complete, whereas the desorption of $\mathrm{Cu}^{2+}$ is partial. $50 \%$ of the metal ions remain on the resin at equilibrium. Thus, the $P A A 20^{\prime} 000$ is slightly more efficient than the PEIAC 25'000 for the desorption of $C u^{2+}$.

The influence of the molecular mass of the complexing agent has been studied for the desorption of $\mathrm{Ni}^{2+}$. Fig. 4 shows a typical desorption of $\mathrm{Ni}^{2+}$ with the PEIAC 750'000 (REG148). The desorption of $\mathrm{Ni}^{2+}$ is partial. The equilibrium is not totally reached after an elution volume $V_{E}=300 \mathrm{ml}$, because there is a kinetic effect. The remainder of the metal ions at equilibrium, reached after an elution volume $V_{E}=600$ $\mathrm{ml}$, represents $13 \%$ of the initial amount.

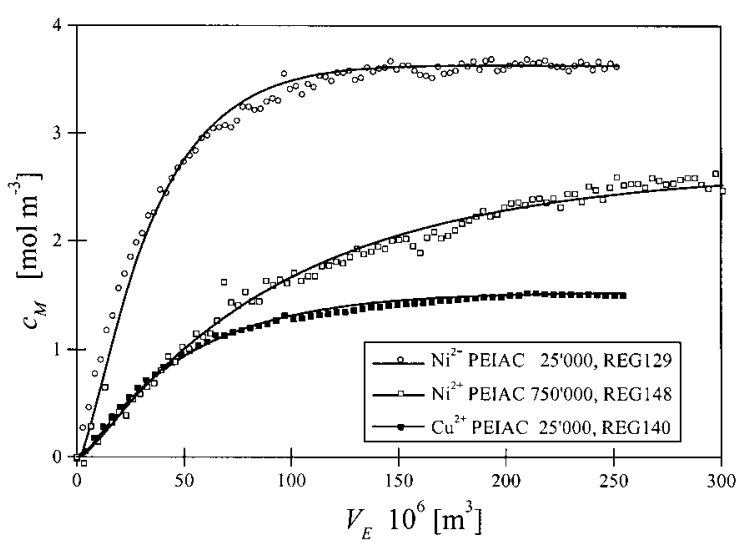

Figure 4. Effect of the type of metal $\left(\mathrm{Ni}^{2+}\right.$ and $\left.\mathrm{Cu}^{2+}\right)$ on the desorption of the resin Chelamine with PEIAC 25'000. $m=0.4 \mathrm{~g}, \mathrm{pH}=7.3$, simplified model for $\mathrm{Ni}^{2+}$. Desorption of $\mathrm{Ni}^{2+}$ from the resin Chelamine with PEIAC 750'000. $m=0.4$ $\mathrm{g}, \mathrm{pH}=7.3$.

The constant $K_{1}$ for $P E I A C 750$ '000 is lower than for PEIAC $25^{\prime} 000$ and this latter than for $P A A 20^{\prime} 000$. The parameter $k_{2}$ is smaller for $\mathrm{Cu}^{2+}$ than for $\mathrm{Ni}^{2+}$ and changes slightly when the simplified model is used (Tabs. 2 and 3). The values for $K_{2}$ describing the second equilibrium show that the systems PEIAC $25^{\prime} 000 / \mathrm{Cu}^{2+}$ or PAA $20^{\prime} 000 / \mathrm{Cu}^{2+}$ are less favorable than the system PEIAC 750 '000/Ni ${ }^{2+}$, thus the quantity of remaining metal ions on the resin at equilibrium is greater for the first two systems. The results led to the following conclusions:

- For a given polymer and a given molecular mass, the parameter $K_{1}$ remains identical.

- The parameter $k_{2}$ depends on the type of metal.

\subsection{Simultaneous Desorption of the Resin Loaded with $\mathrm{Cu}^{2+}$ and $N i^{2+}$}

A simultaneous desorption of $\mathrm{Cu}^{2+}$ and $\mathrm{Ni}^{2+}$ with PEIAC $25^{\prime} 000$ (REG143) is presented in this section. The resin was loaded with an almost equimolar solution of $\mathrm{Cu}^{2+}$ and $\mathrm{Ni}^{2+}$, therefore the resin was saturated as there was an excess of metal ions.

$N i^{2+}$ is totally desorbed (analytical limit) while a large portion of $\mathrm{Cu}^{2+}(70 \%)$ is still in equilibrium with the resin and the polymer. Fig. 5 gives the desorption concentrations profiles of both metal ions in the CSTR. The small absorbance of the complex PEIAC/Ni $i^{2+}$ explains the poor quality of the $\mathrm{Ni}^{2+}$ desorption curve.

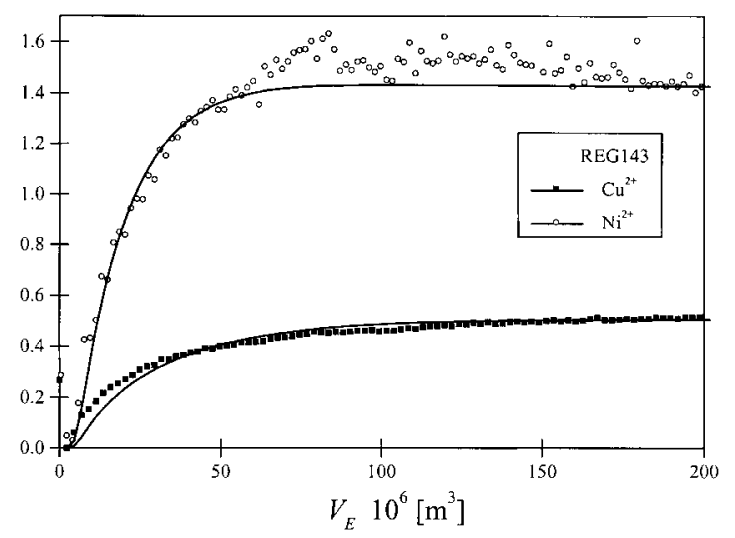

Figure 5. Simultaneous desorption of $\mathrm{Cu}^{2+}$ and $\mathrm{Ni2}+$ from the resin Chelamine with PEIAC 25'000. $c_{P, 0}=33.51 \mathrm{~mol} \mathrm{~m}^{-3}, m=0.4 \mathrm{~g}, \mathrm{pH}=7.3, \gamma=8.81, K_{1}=0.035$ $\mathrm{m}^{3} \mathrm{~mol}^{-1}, k_{2, C u}=2.110^{-3} \mathrm{~s}^{-1}, k_{-2, C u}=5.710^{-3} \mathrm{~m}^{3} \mathrm{~s}^{-1} \mathrm{~mol}^{-1}, k_{2, N i}=8.010^{-3} \mathrm{~s}^{-1}$, simplified model for $\mathrm{Ni}^{2+}$.

The application of (7a) to the simultaneous desorption of $\mathrm{Cu}^{2+}$ and $\mathrm{Ni}^{2+}$ requires the introduction of mass balances (in each part of the reactor) and an additional kinetics equation for the second 
metal. The same equilibrium $K_{1}$ is applied to both kinetic equations, as this constant does not depend on the type of metal. The model applied to the desorption of $\mathrm{Cu}^{2+}$ and $\mathrm{Ni}^{2+}$ includes four parameters: $K_{1}, k_{2, C u}, k_{-2, C u}$ and $k_{2, N i}$. The mean parameters determined separately for each metal without any optimization are used for the simulation (Tab. 3). A correction on constant $k_{-2, C u}$ (by a factor 7) is necessary to obtain a correct description. This can be explained by the competition of desorption due to different kinetics of desorption of both metal.

\subsection{Validation of the Model for a Pilot Fixed Bed}

The purpose of this section is to show the validity of the simplified kinetic model at a scale 40 times bigger. The values and operating conditions used are summarized in Tab. 4.

Fig. 6 shows the pilot desorption of $\mathrm{Ni}^{2+}$ from the resin Chelamine with PEIAC 25'000. The parameters used for the calculations were those obtained at laboratory scale for the simplified model $\left(K_{1}=0.035 \mathrm{~m}^{3} \mathrm{~mol}^{-1}, k_{2}=8.010^{-3} \mathrm{~s}^{-1}\right)$ without adjustment, as well as the measured values for flow rate, resin capacity and initial concentration of the polymer free sites.

The result for the pilot desorption shows that the desorption kinetics remains valid at mini-pilot scale. The metal ions still fixed on the resin $\left(q_{\text {rest }}=0.065 \mathrm{~mol} \mathrm{~kg}^{-1}\right)$ do not induce any problem in the global regeneration process as these correspond to less than $8 \%$ of the total desorbed metal ions. This experiment proves that valid calculated simulations can be obtained if one of the experimental parameters is changed. The molar ratio between the $\mathrm{NaOH}$ added in the CSTR and the desorbed $\mathrm{Ni}^{2+}$ is also analyzed. A value of 1.97 is obtained for the pilot desorption. If the added base compensates the decrease in $\mathrm{pH}$ due to desorption, the complexation mechanism between the metal ions and the polymer proposed by Geckeler et al. [8] is confirmed, i.e., the adsorption of each metallic divalent cation to the polymer discharges two protons $H^{+}$.

\section{Conclusions}

The new regeneration system presented in this study is part of a global process of regeneration of heavy-metal-loaded chelating resins [3]. The global process consists of a coupling of the adsorption of the heavy-metal ions in a fluidized bed, desorption with soluble polymer in a fixed bed, reverse osmosis or ultrafiltration to concentrate the desorption solution of the complex polymer/ $\mathrm{M}^{2+}$ and electrolysis of the solution to recover the metal.

A complete model describing the kinetics of desorption of heavy-metal-loaded resin has been proposed. It allows the description of partial desorption and can be simplified for a total desorption. The variation of operating conditions, as well

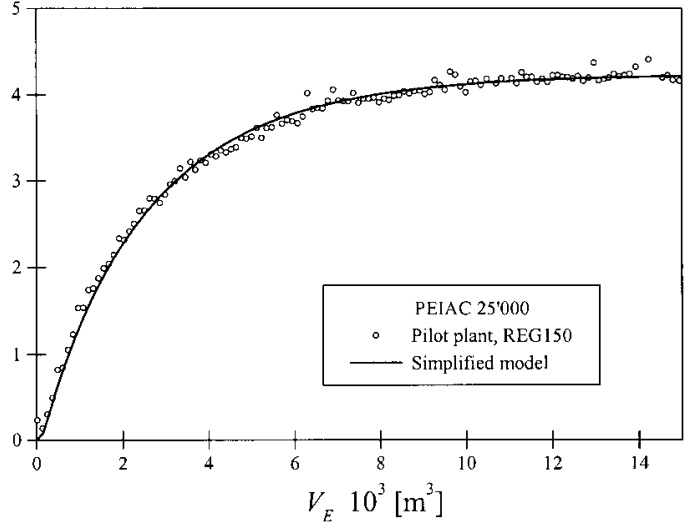

Figure 6. Pilot desorption of $\mathrm{Ni}^{2+}$ from the resin Chelamine with PEIAC 25'000. $c_{P, 0}=10.38 \mathrm{~mol} \mathrm{~m}^{-3}, m=17.05 \mathrm{~g}, \mathrm{pH}=7.3, K_{1}=0.035 \mathrm{~m}^{3} \mathrm{~mol}^{-1}, k_{2}=8.010^{-3} \mathrm{~s}^{-1}$, $Q=3.39510^{-6} \mathrm{~m}^{3} \mathrm{~s}^{-1}, r=0.1$, simplified model.

as the types of complexing agents and heavy-metal ions, has shown that the desorption can be described in all cases studied. The kinetic model was used successfully for a scale-up. In the global process of heavy metal recovery, this semiclosed desorption allows almost total recycling of the desorption agent and avoids large consumption of chemicals, as is the case for classical acid-base regeneration. The kinetic study reported in this paper leads to the following conclusions:

1) The type of complexing agent dictates the first equilibrium.

The $K_{1}$ constant represents the strength of the complexing agent with regard to the desorption.

2) The second equilibrium (or the reaction described by the constant $k_{2}$ for the simplified model) depends on the type of heavy metal. The desorption is not complete for the systems PEIAC 25'000/Cu ${ }^{2+}$, PEIAC 750'000/ $\mathrm{Ni}^{2+}$ and $P A A 20^{\prime} 000 / C u^{2+}$, and a final equilibrium is reached.

From the presented results, it can be concluded that the method proposed is simple and effective for the removal of metal ions from dilute solutions and for an efficient desorption of loaded chelating resins with strong complexing agents.

\section{Acknowledgements}

This research is supported by the Swiss Commission for Technology and Innovation (CTI). The authors would like to thank Dr G. Jeanneret (JPS-Chimie, Neuchâtel, Switzerland) 
for the synthesis of the resin Chelamine Standard and the soluble polymers PEIAC.

Received: July 7, 1999 [CET 1134]

\section{Symboles used}

\begin{tabular}{|c|c|c|}
\hline$c_{P, i}$ & {$\left[\mathrm{~mol} \mathrm{~m}^{-3}\right]$} & $\begin{array}{l}\text { free polymer sites concentration in the } \\
\text { tank } i \text { of the fixed bed }\end{array}$ \\
\hline$c_{P, 0}$ & {$\left[\mathrm{~mol} \mathrm{~m}^{-3}\right]$} & $\begin{array}{l}\text { initial free polymer sites concentration } \\
\text { in the CSTR }\end{array}$ \\
\hline$c_{0}$ & {$\left[\mathrm{~kg} \mathrm{~m}^{-3}\right]$} & $\begin{array}{l}\text { metal ion concentration of the loading } \\
\text { solution }\end{array}$ \\
\hline$c_{M, i}$ & {$\left[\mathrm{~mol} \mathrm{~m}^{-3}\right]$} & $\begin{array}{l}\text { concentration of the polymer- } \\
\text { complexed sites } P M^{2+} \text { in the tank } i \text { of } \\
\text { the fixed bed }\end{array}$ \\
\hline$d_{t}$ & {$[\mathrm{~m}]$} & column diameter \\
\hline$k_{1}$ & {$\left[\mathrm{~m}^{3} \mathrm{~mol}^{-1} \mathrm{~s}^{-1}\right]$} & kinetic constant \\
\hline$k_{-1}$ & {$\left[\mathrm{~s}^{-1}\right]$} & kinetic constant \\
\hline$k_{2}$ & {$\left[\mathrm{~s}^{-1}\right]$} & kinetic constant \\
\hline$k_{-2}$ & {$\left[\mathrm{~m}^{3} \mathrm{~mol}^{-1} \mathrm{~s}^{-1}\right]$} & kinetic constant \\
\hline$K_{1}$ & {$\left[\mathrm{~m}^{3} \mathrm{~mol}^{-1}\right]$} & equilibrium constant \\
\hline$K_{2}$ & {$\left[\mathrm{~mol} \mathrm{~m}^{-3}\right]$} & equilibrium constant \\
\hline$m$ & {$[\mathrm{~kg}]$} & mass of resin in the reactor \\
\hline$M_{w}$ & {$\left[\mathrm{~g} \mathrm{~mol}^{-1}\right]$} & mass molecular weight \\
\hline$N$ & {$[-]$} & number of stirred tanks in the fixed bed \\
\hline$Q$ & {$\left[\mathrm{~m}^{3} \mathrm{~s}^{-1}\right]$} & flow rate \\
\hline $\bar{q}_{P}$ & {$\left[\mathrm{~mol} \mathrm{~kg}^{-1}\right]$} & polymer capacity \\
\hline$q_{c, i}$ & {$\left[\mathrm{~mol} \mathrm{~kg}^{-1}\right]$} & $\begin{array}{l}\text { metal ion concentration in the } \\
\text { intermediary form }\left\{R M^{2+^{P l}} \text { in the tank } i\right. \\
\text { of the fixed bed }\end{array}$ \\
\hline$q_{d e s}$ & {$\left[\mathrm{~mol} \mathrm{~kg}^{-1}\right]$} & concentration of desorbed metal \\
\hline$q_{i}$ & {$\left[\mathrm{~mol} \mathrm{~kg}^{-1}\right]$} & $\begin{array}{l}\text { sum of the metal ion concentrations on } \\
\text { the resin } R M^{2+} \text { and in the form of } \\
\text { intermediary }\left\{R M^{2+} P\right\} \text { in the tank } i \text { of } \\
\text { the fixed bed }\end{array}$ \\
\hline$q_{\text {rest }}$ & {$\left[\mathrm{mol} \mathrm{kg}^{-1}\right]$} & $\begin{array}{l}\text { metal ion concentration remaining on } \\
\text { the resin }\end{array}$ \\
\hline$q_{0}$ & {$\left[\mathrm{~mol} \mathrm{~kg}^{-1}\right]$} & $\begin{array}{l}\text { initial metal ion concentration on the } \\
\text { resin (in equilibrium with } \\
\text { concentration } c_{O} \text { ) }\end{array}$ \\
\hline$r_{p}$ & {$[\mathrm{~m}]$} & mean radius of the particles \\
\hline$r$ & {$[-]$} & $\begin{array}{l}\text { fraction of flow rate going through the } \\
\text { dead volume }\end{array}$ \\
\hline$t$ & {$[\mathrm{~s}]$} & time \\
\hline$V$ & {$\left[\mathrm{~m}^{3}\right]$} & volume of the reactor \\
\hline$V_{E}$ & {$\left[\mathrm{~m}^{3}\right]$} & $\begin{array}{l}\text { volume of liquid passed through the } \\
\text { fixed bed }\end{array}$ \\
\hline
\end{tabular}

$\begin{array}{lr}V_{M} & {\left[\mathrm{~m}^{3}\right]} \\ V_{C} & {\left[\mathrm{~m}^{3}\right]} \\ V_{C, O} & {\left[\mathrm{~m}^{3}\right]} \\ V_{\mathrm{NaOH}} & {\left[\mathrm{m}^{3}\right]}\end{array}$

Greek letters

$\begin{array}{ll}\gamma & {[-]} \\ \varepsilon_{P M, \lambda} & {\left[\mathrm{M}^{-1} \mathrm{~cm}^{-1}\right]} \\ & \\ \varepsilon_{r} & {[-]} \\ \lambda & {[\mathrm{nm}]} \\ \tau & {[\mathrm{s}]}\end{array}$

\section{Abbreviations}

$\begin{array}{ll}\text { CSTR } & \text { continuous stirred tank reactor } \\ E D T A & \text { ethylenediamine tetraacetate } \\ M^{2+} & \text { divalent metallic cation } \\ N T A & \text { nitrilotriacetate } \\ P & \text { polymer free site } \\ P A A & \text { polyacrylic acetate } \\ P E I A C & \text { polyethyleneimineacetic acetate } \\ P M^{2+} & \text { complexed-polymer site } \\ R & \text { resin-free site } \\ R M^{2+} & \text { resin site loaded with metal ions } \\ \left\{R M^{2+} P\right\} & \text { intermediary complex }\end{array}$

\section{References}

[1] Menoud, P.; Cavin, L; Renken, A., Chem. Eng. Proc. 37 (1998) pp. 89-101.

[2] Menoud, P., Ph. D. Thesis, EPF Lausanne 1996.

[3] Menoud, P.; Schmidt, V.; Meyer, T.; Renken, A., Selective Separation and Recovery of Heavy Metals from Industrial Wastewater, Proceedings of the Int. Congress on Recovery Recycling Reintegration (R'95), Geneva, 3 (1995) pp. 378-384.

[4] Bolto, B. A.; Eldridge, R. J.; Kotowski, M.; Pawlowski, M.; Swinton, E. A.; Wasag, H., React. Pol. 2 (1984) pp. 223-231.

[5] Tipnis, U. K.; Dasare, B. D., Indian Environ. Prot. 8 (1998) No. 12, No. 914 918.

[6] Geckeler, K.; Weingärtner, K.; Bayer, E., Metal Complexation of Poly(vinylamine) and Derivatives, Polymeric Amines an Contributed Papers, Int. Symposium, Ghent 1979, pp. 277-285.

[7] Shriver, D. F.; Atkins, P. W.; Langford, C. H., Inorganic Chemistry, Oxford University Press, Oxford 1992.

[8] Geckeler, K.; Lange, G.; Eberhardt, H.; Bayer, E., Pure Appl. Chem. 52 (1980) pp. 1883-1905.

[9] Schumann, G., Chem. Tech. 43 (1991) No. 7, pp. 254-257. 